\title{
Filters of residuated lattices and triangle algebras
}

\author{
B. Van Gasse ${ }^{1}$, G. Deschrijver, C. Cornelis ${ }^{1}$, E. E. Kerre \\ Fuzziness and Uncertainty Modelling Research Unit \\ Department of Applied Mathematics and Computer Science \\ Ghent University, Krijgslaan 281 (S9), 9000 Gent, Belgium
}

\begin{abstract}
An important concept in the theory of residuated lattices and other algebraic structures used for formal fuzzy logic, is that of a filter. Filters can be used, amongst others, to define congruence relations. Specific kinds of filters include Boolean filters and prime filters.

In this paper, we define several different filters of residuated lattices and triangle algebras and examine their mutual dependencies and connections. Triangle algebras characterize interval-valued residuated lattices.
\end{abstract}

Key words:

filters, interval-valued structures, residuated lattices

1991 MSC: 03B45, 03B47, 03B50, 03B52, 06F05, 08A72

\section{Introduction and preliminaries}

Filters are subsets of partially ordered sets that satisfy some defining properties. The concept is also used in partially ordered algebraic structures with more connectives (lattices, residuated lattices, MTL-algebras,... ), in particular in the algebraic semantics of formal fuzzy logics. Filters are of crucial importance in the proof of the (chain) completeness of these logics. Filters are also particularly interesting because they are closely related to congruence

Email addresses: Bart.VanGasse@UGent. be (B. Van Gasse), Glad.Deschrijver@UGent.be (G. Deschrijver), Chris.Cornelis@UGent. be (C. Cornelis), Etienne.Kerre@UGent.be (E. E. Kerre).

1 Bart Van Gasse and Chris Cornelis would like to thank the Research FoundationFlanders for funding their research. 
relations; with every filter we can associate a quotient algebra. If we want such a quotient algebra to satisfy particular properties, we need to put extra conditions on the filter. These conditions are often of the form " $f\left(x_{1}, \ldots, x_{n}\right)$ must be an element of the filter for all $x_{1}, \ldots, x_{n}$ in the algebraic structure". In bounded partially ordered sets at most one filter can be a singleton, namely $\{1\}$. Stating that this singleton is a specific kind of filter, is equivalent with requiring the algebraic structure to fulfill a particular property. For example, $\{1\}$ is a prime filter of a residuated lattice if, and only if, that residuated lattice is linear (i.e., totally ordered). In this way, we can translate connections between properties on algebraic structures to connections between properties of the filter $\{1\}$. Often these connections can be generalized to any filter (not necessarily $\{1\}$ ). We will give several examples in Section 2 .

Prime filters are also interesting because they can be used to prove that MTLalgebras are subdirect products of linear residuated lattices.

In this paper ${ }^{2}$, a residuated lattice is defined as a structure $\mathcal{L}=(L, \sqcap, \sqcup, *, \Rightarrow$, 0,1 ) in which $\sqcap, \sqcup, *$ and $\Rightarrow$ are binary operators (called infimum, supremum, product and implication) on $L$ satisfying:

- $(L, \sqcap, \sqcup)$ is a bounded lattice with 0 as smallest and 1 as greatest element,

- * is commutative and associative, with 1 as neutral element, and

- $x * y \leq z$ iff $x \leq(y \Rightarrow z)$ for all $x, y$ and $z$ in $L$ (residuation principle).

The ordering $\leq$ and negation $\neg$ in a residuated lattice $\mathcal{L}=(L, \sqcap, \sqcup, *, \Rightarrow, 0,1)$ are defined as follows, for all $x$ and $y$ in $L: x \leq y$ iff $x \sqcap y=x$ (or equivalently, iff $x \sqcup y=y$; or, also equivalently, iff $x \Rightarrow y=1$ ) and $\neg x=x \Rightarrow 0$.

An element $l$ of $L$ is idempotent iff $l * l=l$, it is nilpotent iff there exists an integer $n$ such that $\underbrace{x * x * \ldots * x}_{n \text { times }}=0$.

Proposition 1 In a residuated lattice $(L, \sqcap, \sqcup, *, \Rightarrow, 0,1)$, * is increasing in both arguments, $\Rightarrow$ only in the second. In the first argument, $\Rightarrow$ is decreasing. In particular, $\neg$ is a decreasing operator. Furthermore the following inequalities and identities hold, for every $x, y$ and $z$ in $L$ :

(1) $x * y \leq x \sqcap y$,

(2) $\neg x \sqcup y \leq x \Rightarrow y$,

(3) $x * \neg y \leq \neg(x \Rightarrow y)$,

(4) $x \leq y \Rightarrow(x * y)$,

(5) $x *(x \Rightarrow y) \leq x \sqcap y$ (in particular: $x * \neg x=0)$,

(6) $x \sqcup y \leq(x \Rightarrow y) \Rightarrow y$ (in particular: $x \leq \neg \neg x)$,

$\overline{2}$ In literature (e.g. in [12]), the name residuated lattice is sometimes used for more general structures than what we call residuated lattices. In the most general terminology, our structure would be called a bounded integral commutative residuated lattice. 
(7) $\neg \neg \neg x=\neg x$,

(8) $(x \Rightarrow y) * z \leq x \Rightarrow(y * z)$,

(9) $x \Rightarrow(y \sqcap z)=(x \Rightarrow y) \sqcap(x \Rightarrow z)$,

(10) $(x \sqcup y) \Rightarrow z=(x \Rightarrow z) \sqcap(y \Rightarrow z)$ (in particular: $\neg(x \sqcup y)=\neg x \sqcap \neg y)$,

(11) $(x * y) \Rightarrow z=x \Rightarrow(y \Rightarrow z)$ (in particular: $\neg(x * y)=x \Rightarrow \neg y)$,

(12) $x \Rightarrow y$ is equal to the largest element $z$ in $L$ that satisfies $x * z \leq y$, so we have $x \Rightarrow y=\sup \{z \in L \mid x * z \leq y\}$ (in particular: $1 \Rightarrow y=y$ )

(13) $x *(y \sqcup z)=(x * y) \sqcup(x * z)$,

(14) $(x \Rightarrow y) *(y \Rightarrow z) \leq x \Rightarrow z$

(15) $x \Rightarrow y \leq(x * z) \Rightarrow(y * z)$.

The proofs can be found in, e.g., [23].

Residuated lattices [2] form a variety and constitute the semantics of Höhle's Monoidal Logic (ML) [12], which is the basis for the majority of formal fuzzy logics, like Esteva and Godo's Monoidal T-norm based Logic (MTL) [5], Hájek's Basic Logic (BL) [10], Łukasiewicz Logic (LL) [18], Intuitionistic Logic (IL) [11] and Gödel Logic (GL) [3,8]. For other examples, a good overview and more details, we refer to $[6,9,10]$.

An MTL-algebra [5] is a prelinear residuated lattice, i.e., a residuated lattice in which $(x \Rightarrow y) \sqcup(y \Rightarrow x)=1$ for all $x$ and $y$ in $L$. Linear residuated lattices are always prelinear. In linear residuated lattices, 1 is always $\sqcup$-irreducible. This means that for all $x$ and $y$ in $L, x \sqcup y=1$ iff $x=1$ or $y=1$ (or both). A Heyting-algebra, or pseudo-Boolean algebra [22], is a residuated lattice in which $x * x=x$ for all $x$ in $L$, or, equivalently, in which $x * y=x \sqcap y$ for all $x$ and $y$ in $L$.

A Boolean algebra [13] is a residuated lattice in which $x \sqcup \neg x=1$ for all $x$ in $L$. It is always a Heyting-algebra and prelinear.

Definition 2 [10,12,15-17,21,24] A filter of a residuated lattice $\mathcal{L}=(L, \sqcap, \sqcup$, $*, \Rightarrow, 0,1)$ is a non-empty subset $F$ of $L$ satisfying

- for all $x$ and $y$ in $L$ : if $x \in F$ and $x \leq y$, then $y \in F$,

- for all $x$ and $y$ in $F: x * y \in F$ (i.e., $F$ is closed under $*$ ).

$A$ Boolean filter $(B F)$ of $\mathcal{L}$ is a filter $F$ satisfying

- for all $x$ in $L: x \sqcup \neg x \in F$.

A prime filter $(P F)$ of $\mathcal{L}$ is a filter $F$ satisfying

- for all $x$ and $y$ in $L: x \Rightarrow y \in F$ or $y \Rightarrow x \in F$ (or both).

A prime filter of the second kind (PF2) is a filter F satisfying 
- for all $x$ and $y$ in $L:$ if $x \sqcup y \in F$, then $x \in F$ or $y \in F$ (or both).

A positive implicative filter of $\mathcal{L}$ is a subset $F$ of $L$ such that

- $1 \in F$,

- for all $x, y$ and $z$ in $F:$ if $(x * y) \Rightarrow z \in F$ and $x \Rightarrow y \in F$, then $x \Rightarrow z \in F$.

An alternative definition for a filter $F$ (see e.g. [23]) of a residuated lattice $\mathcal{L}=(L, \sqcap, \sqcup, *, \Rightarrow, 0,1)$ is the following:

- $1 \in F$,

- for all $x$ and $y$ in $L$ : if $x \in F$ and $x \Rightarrow y \in F$, then $y \in F$.

An alternative definition for a Boolean filter $F$ of a residuated lattice $\mathcal{L}=$ $(L, \sqcap, \sqcup, *, \Rightarrow, 0,1)$ is the following: $F$ is a filter of $\mathcal{L}$ satisfying

- for all $x, y$ and $z$ in $L$ : if $(x * \neg z) \Rightarrow y \in F$ and $y \Rightarrow z \in F$, then $x \Rightarrow z \in F$.

This was proven in [17], where these filters were called implicative filters. It was also shown in [17] that a Boolean filter is always a positive implicative filter and that also the converse is true in residuated lattices with an involutive negation $\neg$. A positive implicative filter of a residuated lattice is always a filter of that residuated lattice. [17]

\section{$2 \quad$ Filters of residuated lattices}

\subsection{Finite filters and finite prime filters of the second kind}

If $\mathcal{L}=(L, \sqcap, \sqcup, *, \Rightarrow, 0,1)$ is a residuated lattice and $l \in L$ such that $l * l=l$, then it is not difficult to see that $F_{l}:=\{x \in L \mid l \leq x\}$ is a filter of $\mathcal{L}$. In fact, finite filters of residuated lattices are always of this form.

Proposition 3 Let $\mathcal{L}=(L, \sqcap, \sqcup, *, \Rightarrow, 0,1)$ be a residuated lattice and $F$ a finite filter of $\mathcal{L}$. Then $F=F_{l}$ for some idempotent $l$ in $L$.

PROOF. If $F$ is a finite filter of $\mathcal{L}$, then the product $m$ of all its elements (a finite number) must also be an element of $F$. So $m * m \in F$ and therefore $m$ (as the product of all elements of $F$ ) is smaller than or equal to $m * m$ (because of Proposition 1(1)), which implies $m * m=m$.

Remark that this property does not hold for infinite filters. For example, ]0, 1] is a filter of $\left([0,1], \min , \max , \min , \Rightarrow_{\min }, 0,1\right)$, but $\left.] 0,1\right]$ is not of the form $F_{l}$ 
for any $l \in[0,1]$.

Finite prime filters of the second kind of residuated lattices are always of the form $F_{l}$, with $l$ idempotent and $\sqcup$-irreducible. Conversely, filters (even infinite ones) of this form will always be prime filters of the second kind.

Proposition 4 Let $\mathcal{L}=(L, \sqcap, \sqcup, *, \Rightarrow, 0,1)$ be a residuated lattice. If $l$ is an idempotent and $\sqcup$-irreducible element of $L$, then $F_{l}$ is a prime filter of the second kind of $\mathcal{L}$.

PROOF. Suppose $a \sqcup b \in F_{l}$. Then $l \leq a \sqcup b$ and therefore, using Proposition $1(13), l=l * l \leq l *(a \sqcup b)=(l * a) \sqcup(l * b) \leq l$. So $l=(l * a) \sqcup(l * b)$, which implies $l=l * a$ or $l=l * b$. So $l \leq a$ or $l \leq b$, which means exactly that $a \in F_{l}$ or $b \in F_{l}$.

\subsection{Boolean filters of the second kind and prime filters of the third kind}

Some particular kinds of filters appear quite frequently in our investigation of the connections between different kinds of filters. Therefore we gave them a name and introduce them in the next definition.

Definition 5 A Boolean filter of the second kind (BF2) of a residuated lattice $\mathcal{L}=(L, \sqcap, \sqcup, *, \Rightarrow, 0,1)$ is a filter of $\mathcal{L}$ satisfying

- for all $x$ in $L: x \in F$ or $\neg x \in F$ (or both).

A prime filter of the third kind (PF3) of a residuated lattice $\mathcal{L}=(L, \sqcap, \sqcup, *, \Rightarrow$, $0,1)$ is a filter of $\mathcal{L}$ satisfying

- for all $x$ and $y$ in $L:(x \Rightarrow y) \sqcup(y \Rightarrow x) \in F$.

It can easily be seen there is always a trivial example of each kind of filter defined before, namely $L$; and that every filter always contains the greatest element 1.

Definition 6 Let $S$ be a set and $\mathcal{C}$ be a subset of its powerset $P(S)$.

$W e$ say $\mathcal{C}$ satisfies the intersection property iff $\mathcal{C}$ is closed under intersections. $W e$ say $\mathcal{C}$ satisfies the monotonicity property iff $\mathcal{C}$ is closed under supersets, i.e., iff $S_{2} \in \mathcal{C}$ whenever $S_{1} \in \mathcal{C}$ and $S_{1} \subseteq S_{2} \subseteq S$.

Straightforward to verify is that filters, prime filters of the third kind and Boolean filters satisfy the intersection property. We will see later that it also holds for positive implicative filters. For PF, PF2 and BF2 the intersection property does not hold, as will be shown in some of the examples. 
From the definitions it is also clear that filters, prime filters, prime filters of the third kind, Boolean filters and Boolean filters of the second kind satisfy the monotonicity property. In [17] it was shown that it also holds for positive implicative filters. In Example 7 we will show that the monotonicity property does not hold for prime filters of the second kind. ${ }^{3}$ Remark that the monotonicity property suggests a special role for the singleton $\{1\}$, which is always a filter. Indeed, in order to show that all filters in a residuated lattice $\mathcal{L}$ are $\mathrm{PF}$ (PF3, BF or BF2, resp.), it suffices to show that $\{1\}$ is $\mathrm{PF}$ (PF3, BF or BF2, resp.). Therefore we first take a closer look at the filter $\{1\}$. The proofs immediately follow from the definitions.

- It is a Boolean filter iff $\mathcal{L}$ is a Boolean algebra. In this case, all filters are Boolean filters.

- It is a Boolean filter of the second kind iff $\mathcal{L}$ is the Boolean algebra with two elements (or the trivial Boolean algebra with one element). In this case, all filters are Boolean filters of the second kind.

- It is a prime filter iff $\mathcal{L}$ is linearly ordered. In this case, all filters are prime filters.

- It is a prime filter of the second kind iff 1 is $\sqcup$-irreducible in $\mathcal{L}$. Remark that in general, this does not imply that all filters are prime filters of the second kind. A counterexample will be given in Example 7.

- It is a prime filter of the third kind iff $\mathcal{L}$ is an MTL-algebra. In this case, all filters are prime filters of the third kind.

- It is a positive implicative filter iff ${ }^{4} \mathcal{L}$ is a Heyting-algebra. In this case, all filters are positive implicative filters.

Now, before we further investigate the connections between the different kinds of filters, we first give some easy but useful examples ${ }^{5}$.

\subsection{Examples of filters of residuated lattices}

Example 7 Three residuated lattices exist on the lattice in Figure 1 (5.1.20 in [14]). If we consider the Heyting-algebra, the filters are $\{1\},\{v, 1\},\{a, v, 1\}$, $\{b, v, 1\}$ and $\{0, a, b, v, 1\}$. Remark that $\{1\}$ is PF2, but $\{v, 1\}$ is not. So the monotonicity property does not hold for PF2. Also note that $\{1\}$ is not PF3, as prelinearity does not hold: $(a \Rightarrow b) \sqcup(b \Rightarrow a)=b \sqcup a=v$. The filters $\{a, v, 1\}$, $\{b, v, 1\}$ and $\{0, a, b, v, 1\}$ are PF, PF2, PF3, BF and BF2. Note however that

$\overline{3}$ Another reason why PF2 can be regarded as the odd one out amongst PF, PF2, $\mathrm{PF} 3, \mathrm{BF}$ and BF2, is that the condition 'if $x \sqcup y \in F$, then $x \in F$ or $y \in F$ ' in its definition only depends on the lattice structure, not on the product or implication.

4 This follows from the equivalence between (1) and (4) in Proposition 16.

5 The small examples can be found in [14]. This website contains all residuated lattices (according to the most general definition) up to size 6 . 


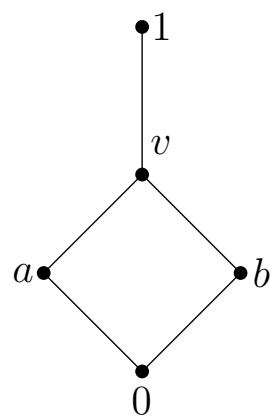

Fig. 1. Doubleton $\{v, 1\}$ is a filter of the Heyting-algebra on this lattice, but not a prime filter of the second kind. The singleton $\{1\}$ is a prime filter of the second kind, but not a prime filter.

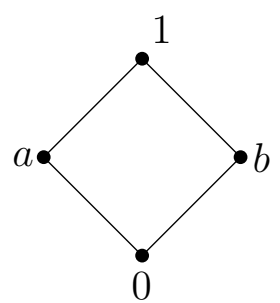

Fig. 2. A Boolean algebra in which 1 is not $\sqcup$-irreducible.

their intersection $\{v, 1\}$ is not PF, PF2 nor BF2. So the intersection property does not hold for PF, PF2 and BF2.

Example 8 Only one residuated lattice exists on the lattice displayed in Figure 2 (4.1.5 in [14]). This is a Boolean algebra (isomorphic to the powerset $\mathcal{P}(\{a, b\})$ endowed with intersection and union). So $\{1\}$ is a Boolean filter. But it is not a prime filter of the second kind. Similarly as in Example 7, the filters $\{a, 1\},\{b, 1\}$ and $\{0, a, b, 1\}$ are PF, PF2, PF3, BF and BF2, but their intersection $\{1\}$ is not PF, PF2 nor BF2.

Example 9 On a three-element chain (3.1.3 in [14])), there are two residuated lattices. One in which the middle element is idempotent (a Heytingalgebra) and one in which it is nilpotent. In both cases, $\{1\}$ is a prime filter but not a Boolean filter. Remark that there are three filters in the first case and two in the second.

Example 10 Two residuated lattices exist on the lattice in Figure 3 (5.1.17 in [14]): a Heyting-algebra and one in which $v * v=0$. In both cases $\{1\}$ is PF3, but not PF, PF2, BF nor BF2.

Example 11 Seven different residuated lattices exist on the lattice in Figure 4. Exactly one of these is prelinear. In this MTL-algebra, the product $*$ is defined as follows: $x * y=x \sqcap y$ if $x=1, y=1$ or $n \leq x \sqcap y ; x * y=a$ if $\{x, y\}=\{a, c\}$ or $\{x, y\}=\{m, c\} ; x * y=b$ if $\{x, y\}=\{b, d\}$ or $\{x, y\}=$ $\{m, d\}$; and $x * y=0$ otherwise.

One of the six non-prelinear residuated lattices is the Heyting-algebra. The filter $\{1\}$ is not a prime filter of the third kind of this residuated lattice. It is not a prime filter of the second kind either. 


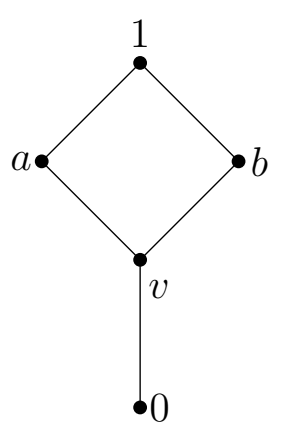

Fig. 3. The residuated lattices on this lattice are prelinear. But they are not Boolean algebras, and 1 is not $\sqcup$-irreducible.

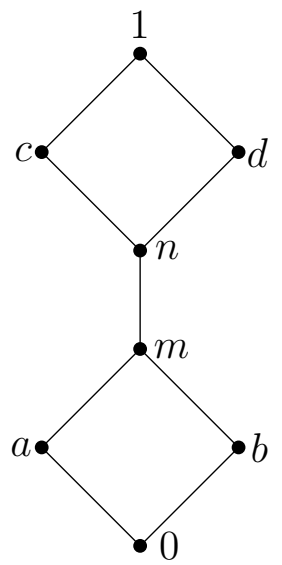

Fig. 4. The Heyting-algebra is not prelinear, and 1 is not $\sqcup$-irreducible.

Remark 12 Prelinearity can be seen as some kind of 'approximation' of linearity (remind that prelinear residuated lattices are subdirect products of linear residuated lattices [5]) and linearity is independent of the product and implication (in other words: if one residuated lattice on a lattice is linear, all residuated lattices on that lattice will be linear). So one might expect that this holds for prelinearity as well. Example 11 shows however that this is not the case, because a prelinear as well as a non-prelinear residuated lattice can exist on the same lattice. Note that for Boolean algebras, we do have such a property. If a Boolean algebra exists on a specific lattice, all residuated lattices on that lattice will be Boolean algebras. To be more precise, there will be only one residuated lattice: the Boolean algebra. The proof of this property is very short. Indeed, suppose $\left(L, \sqcap, \sqcup, \sqcap, \Rightarrow_{\sqcap}, 0,1\right)$ is a Boolean algebra and $\left(L, \sqcap, \sqcup, *, \Rightarrow_{*}, 0,1\right)$ a residuated lattice. Then, because of Proposition 1(1 and 5), for every $x$ in $L$, $x *\left(x \Rightarrow_{\sqcap} 0\right) \leq x \sqcap\left(x \Rightarrow_{\sqcap} 0\right)=0$ and therefore $x \Rightarrow_{\sqcap} 0 \leq x \Rightarrow_{*} 0$. So $1=x \sqcup\left(x \Rightarrow_{\sqcap} 0\right) \leq x \sqcup\left(x \Rightarrow_{*} 0\right)$.

Example 13 There exist thirteen different ${ }^{6}$ residuated lattices on the lattice in Figure 5 (6.1.75 in [14]). None of them is prelinear, so $\{1\}$ is not PF3 in any of them. One of them is determined by $a * a=b * b=u$ and $a * b=0$. The

$\overline{6}$ They are listed in [14] under 6.1.75, together with seven other structures that are not commutative. Three of them are discussed in [6] as well. 


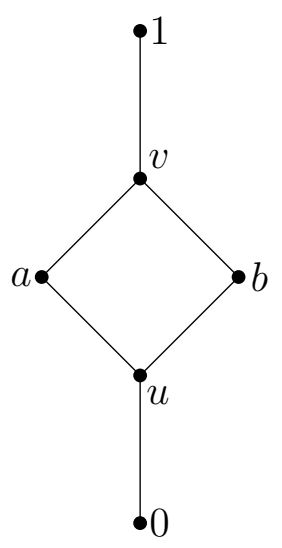

Fig. 5. In some of the residuated lattices on this lattice, all filters are PF2.

filters of this residuated lattice are $\{1\}$ and $\{0, u, a, b, v, 1\}$. Both are prime filters of the second kind.

These examples give us some negative results. The most important ${ }^{7}$ for us are:

- a filter can be PF2, but not PF3; (Example 7)

- a filter can be BF, but not PF2; (Example 8)

- a filter can be PF, but not BF. (Example 9)

Moreover, in each of these three cases, the residuated lattice can be chosen in such a way that $\{1\}$ can serve as the sought-after filter. For example, in Example 9, $\{1\}$ is a prime but not a Boolean filter.

Other negative results are:

- $\{1\}$ being PF2 does not imply all filters being PF2; (Example 7)

- all filters being PF2 does not imply $\{1\}$ being PF3. (Example 13)

Now we continue with the positive results.

\subsection{Connections between the different kinds of filters of residuated lattices}

It is easy to see that a prime filter is always a prime filter of the third kind, and that a Boolean filter of the second kind is always a Boolean filter. The prime filters of a residuated lattice $\mathcal{L}$ are exactly the filters of $\mathcal{L}$ that are

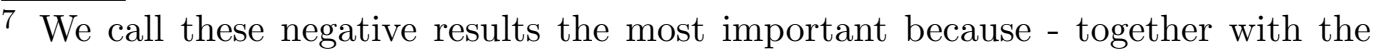
positive results in the next subsection - they imply all negative results amongst the connections between PF, PF2, PF3, BF and BF2. For example, PF3 cannot imply $\mathrm{PF}$ because BF does not imply PF2 and as we will see, BF implies PF3 and PF implies PF2.
} 
at the same time prime filters of the second kind and prime filters of the third kind. This is a corollary of the following proposition.

Proposition 14 Let $\mathcal{L}=(L, \sqcap, \sqcup, *, \Rightarrow, 0,1)$ be a residuated lattice. Every prime filter of $\mathcal{L}$ is also a prime filter of the second kind.

If $\mathcal{L}$ is an $M T L$-algebra, then every prime filter of the second kind of $\mathcal{L}$ is also a prime filter.

PROOF. Suppose $F$ is a prime filter of the residuated lattice $\mathcal{L}=(L, \sqcap, \sqcup, *$, $\Rightarrow, 0,1)$, and $a \sqcup b \in F$. We know that $F$ contains $a \Rightarrow b$ or $b \Rightarrow a$ (or both). Without loss of generality, we assume $a \Rightarrow b \in F$. Then, using Proposition 1(13), $(a *(a \Rightarrow b)) \sqcup(b *(a \Rightarrow b))=(a \sqcup b) *(a \Rightarrow b) \in F$. This implies $b \in F$, because $(a *(a \Rightarrow b)) \sqcup(b *(a \Rightarrow b)) \leq b$ (Proposition 1(5)).

Now suppose $F$ is a prime filter of the second kind of the MTL-algebra $\mathcal{L}=$ $(L, \sqcap, \sqcup, *, \Rightarrow, 0,1)$, and $a, b \in L$. Because $(a \Rightarrow b) \sqcup(b \Rightarrow a)=1 \in F$, either $a \Rightarrow b$ or $b \Rightarrow a$ (or both) must be in $F$.

In residuated lattices that are not MTL-algebras, prime filters of the second kind are in general not prime filters. As a counterexample we can take the filter $\{1\}$ in Example 7 .

In fact, we have not been able to find any non-prelinear residuated lattice in which all prime filters of the second kind are prime filters. Such an example (if it should exist) cannot be finite, due to the next proposition.

Proposition 15 Let $\mathcal{L}=(L, \sqcap, \sqcup, *, \Rightarrow, 0,1)$ be a residuated lattice in which every prime filter of the second kind is also a prime filter. If $L$ is finite or 1 is $\sqcup$-irreducible in $\mathcal{L}$, then $\mathcal{L}$ is prelinear.

PROOF. First note that $\mathcal{L}$ is linear if $|L| \leq 3$, so we only need to consider residuated lattices with 4 elements or more.

If 1 is $\sqcup$-irreducible, then $\{1\}$ is a prime filter of the second kind of $\mathcal{L}$ and by assumption also a prime filter of $\mathcal{L}$. This means that $\mathcal{L}$ is linear (and therefore also prelinear). So in the rest of the proof we can assume $L$ is finite.

Denote $B:=\{x \in L \mid x<1$ and $(\forall y \in L)($ if $x<y$ then $y=1)\}$. In other words, $B$ is the subset of elements of $L$ that are covered by 1 . In the Hassediagram of $\mathcal{L}$, these are the elements that are connected directly with 1 . Because $L$ is finite, $B$ is not empty. We consider two cases:

- $|B|=1$. Say, $B=\{b\}$. Then for all $x$ in $L: x=1$ or $x \leq b$. This implies that 1 is $\sqcup$-irreducible; and we already proved this case.

- $|B|>1$. For any $b \in B$, we will construct a prime filter of the second kind of $\mathcal{L}$ that does not contain $b$. By assumption this filter will be a prime filter of $\mathcal{L}$ and thus also a prime filter of the third kind of $\mathcal{L}$. So for any $b \in B$ 
we obtain a prime filter of the third kind of $\mathcal{L}$ that does not contain $b$. The intersection of these filters is also a prime filter of the third kind of $\mathcal{L}$ and it does not contain any element of $B$. Thus this intersection must be $\{1\}$. Saying that $\{1\}$ is a prime filter of the third kind of $\mathcal{L}$ exactly means that $\mathcal{L}$ is prelinear.

Take any $b$ in $B$. Because $B$ contains at least two elements, we can choose $a_{1} \in B$ such that $b \neq a_{1}$. Note that $b \sqcup a_{1}=1$. If $a_{1}$ is idempotent and $\sqcup$-irreducible, we are done. Because in this case $\left\{a_{1}, 1\right\}$ is a prime filter of the second kind (Proposition 4). If this is not the case, we will show that we can find an element $a_{2}$ in $L$ such that $a_{2}<a_{1}$ and $b \sqcup a_{2}=1$. If $a_{2}$ is idempotent and $\sqcup$-irreducible, we are done. If not, we can find an element $a_{3}$ such that $a_{3}<a_{2}$ and $b \sqcup a_{3}=1$. And so on. Because $L$ is finite, this process must stop at some point. This is only possible if we arrive at an element $a_{k}$ which is idempotent and $\sqcup$-irreducible, giving us the desired prime filter of the second kind, namely $\left\{x \in L \mid a_{k} \leq x\right\}$.

What remains to prove is thus: if $a_{n} \in L$ is such that $b \sqcup a_{n}=1$ and $\left(a_{n} * a_{n}<a_{n}\right.$ or $a_{n}=c \sqcup d$ with $\left.c \neq a_{n} \neq d\right)$, then there exists an element $a_{n+1}$ in $L$ such that $a_{n+1}<a_{n}$ and $b \sqcup a_{n+1}=1$. We have to consider two cases:

(1) $b \sqcup a_{n}=1$ and $a_{n} * a_{n}<a_{n}$. Then we choose $a_{n+1}:=a_{n} * a_{n}$. Indeed, $a_{n+1}<a_{n}$ and (using Proposition 1(1 and 13)) $1=b \sqcup a_{n}=b \sqcup\left(a_{n} *(b \sqcup\right.$ $\left.\left.a_{n}\right)\right)=b \sqcup\left(\left(a_{n} * b\right) \sqcup\left(a_{n} * a_{n}\right)\right)=b \sqcup\left(a_{n} * a_{n}\right)=b \sqcup a_{n+1}$.

(2) $b \sqcup a_{n}=1$ and $a_{n}=c \sqcup d$ with $c \neq a_{n} \neq d$. Suppose $b \sqcup c=b$ and $b \sqcup d=b$. Then $b \sqcup a_{n}=b \sqcup(c \sqcup d)=(b \sqcup c) \sqcup(b \sqcup d)=b<1$, a contradiction. So at least one of $b \sqcup c$ and $b \sqcup d$ must be strictly greater than $b$, and therefore (by definition of $B$ ) equal to 1 , say $b \sqcup c=1$. Then we can choose $a_{n+1}:=c$.

It might be true that MTL-algebras (including all infinite ones) are exactly those residuated lattices in which prime filters and prime filters of the second kind coincide, but this remains an open problem.

Because for any two elements $x$ and $y$ in a residuated lattice it holds that $x \leq y \Rightarrow x$ and $\neg x \leq x \Rightarrow y$ (Proposition 1(2)), it follows that every Boolean filter of the second kind of a residuated lattice is also a prime filter of that residuated lattice, and that every Boolean filter is also a prime filter of the third kind.

Clearly a Boolean filter of the second kind is also any of the other kinds of filters we defined. Remark that any Boolean filter that is at the same time a prime filter of the second kind, is automatically a Boolean filter of the second kind. 


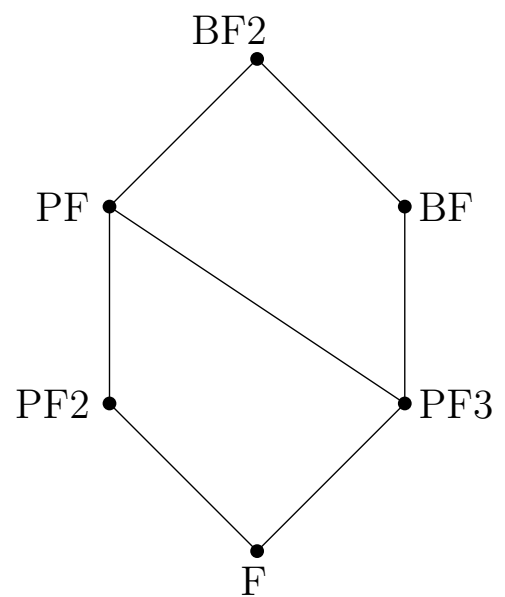

Fig. 6. The six possibilities for a filter.

Considering all examples and all the above implications, we see that only six different situations can occur for a filter $F$ of a residuated lattice $\mathcal{L}$ :

- $F$ is a filter of $\mathcal{L}$, but $F$ is not PF, PF2, PF3, BF nor BF2;

- $F$ is $\mathrm{PF} 2$, but not $\mathrm{PF}, \mathrm{PF} 3, \mathrm{BF}$ nor $\mathrm{BF} 2$;

- $F$ is $\mathrm{PF} 3$, but not $\mathrm{PF}, \mathrm{PF} 2, \mathrm{BF}$ nor $\mathrm{BF} 2$;

- $F$ is PF (and therefore also PF2 and PF3), but not BF nor BF2;

- $F$ is $\mathrm{BF}$ (and therefore also PF3), but not PF, PF2 nor BF2;

- $F$ is BF2 (and therefore also PF, PF2, PF3 and BF).

The filter $\{1\}$ is an example of the first five situations in Examples 11 (the Heyting-algebra), 7, 10, 9 and 8, respectively. It is an example of the last situation in the standard Boolean algebra with two elements.

The lattice in Figure 6 gives a schematic summary of these situations. If $A \leq B$ in this lattice, this means "every filter which is $B$, is also $A$ ". And if $A \sqcup B=C$ in this lattice, this means "every filter which is $A$ as well as $B$, is also $C$ ".

2.5 Positive implicative filters, pseudocomplementation filters and involution filters

In this subsection, we give some alternative characterizations for positive implicative filters and show the connection with the other kinds of filters.

Proposition 16 Let $\mathcal{L}=(L, \sqcap, \sqcup, *, \Rightarrow, 0,1)$ be a residuated lattice and $F$ a filter of $\mathcal{L}$. Then the following statements are equivalent:

(1) $F$ is a positive implicative filter,

(2) $(x \sqcap(x \Rightarrow y)) \Rightarrow y \in F$ for all $x$ and $y$ in $L$,

(3) $(x \sqcap y) \Rightarrow(x * y) \in F$ for all $x$ and $y$ in $L$,

(4) $x \Rightarrow(x * x) \in F$ for all $x$ in $L$. 


\section{PROOF.}

- $(1) \Rightarrow(2)$ : Suppose $F$ is a positive implicative filter of $\mathcal{L}$. Take $x$ and $y$ arbitrarily in $L$. Because $((x \sqcap(x \Rightarrow y)) * x) \Rightarrow y=1 \in F$ and $(x \sqcap(x \Rightarrow$ $y)) \Rightarrow x=1 \in F$ (using Proposition 1(5)), we conclude from the definition of a positive implicative filter that $(x \sqcap(x \Rightarrow y)) \Rightarrow y$ must be in $F$.

- $(2) \Rightarrow(3)$ : For any $x$ and $y$ in $L,(x \sqcap(x \Rightarrow(x * y))) \Rightarrow(x * y)$ is an element of $F$. Because $y \leq x \Rightarrow(x * y),(x \sqcap y) \Rightarrow(x * y)$ is greater than or equal to $(x \sqcap(x \Rightarrow(x * y))) \Rightarrow(x * y)$ and therefore also an element of $F$.

- $(3) \Rightarrow(4)$ : This follows immediately by taking $x=y$.

- $(4) \Rightarrow(1)$ : Choose any $x, y$ and $z$ in $L$ such that $(x * y) \Rightarrow z \in F$ and $x \Rightarrow y \in F$. Then $(x \Rightarrow(x * x)) *(x \Rightarrow y) *((x * y) \Rightarrow z) \in F$. Because (using Proposition 1(15 and 14)) $(x \Rightarrow(x * x)) *(x \Rightarrow y) *((x * y) \Rightarrow z) \leq$ $(x \Rightarrow(x * x)) *((x * x) \Rightarrow(x * y)) *((x * y) \Rightarrow z) \leq x \Rightarrow z$, also $x \Rightarrow z \in F$.

Taking $F=\{1\}$ in Proposition 16 shows that Heyting-algebras are exactly residuated lattices in which $x \sqcap(x \Rightarrow y) \leq y$ for all $x$ and $y$. Or, equivalently, in which $x \sqcap(x \Rightarrow y)=x \sqcap y$ for all $x$ and $y$. This property might be called generalized pseudocomplementation, as the special case $x \sqcap \neg x=0$ is called pseudocomplementation. Another immediate corollary of Proposition 16 is that the intersection and monotonicity property hold for positive implicative filters.

On one hand, Boolean filters (and therefore also Boolean filters of the second kind) are always positive implicative filters. On the other hand, there are no connections with the other kinds of filters we considered:

- a prime filter is not necessarily a positive implicative filter (take for example $\{1\}$ in the residuated lattice with nilpotent middle element in Example 9),

- a prime filter which is also a positive implicative filter is not necessarily a Boolean filter (take for example $\{1\}$ in the residuated lattice with idempotent middle element in Example 9),

- a prime filter of the second kind which is also a positive implicative filter is not necessarily a prime filter of the third kind (take for example $\{1\}$ in Example 7).

If we denote pos. impl. filter, pos. impl. filter + PF2, pos. impl. filter + PF3, and pos. impl. filter $+\mathrm{PF}$ shortly by $\mathrm{A}, \mathrm{B}, \mathrm{C}$ and $\mathrm{D}$, respectively, the situation can be graphically represented as the lattice in Figure 7. At the end of Section 1 we already mentioned that Boolean filters and positive implicative filters of a residuated lattice $\mathcal{L}=(L, \sqcap, \sqcup, *, \Rightarrow, 0,1)$ coincide if $\mathcal{L}$ has an involutive negation. This can be generalized a bit more. First note that positive implicative filters are special cases of pseudocomplementation filters. 


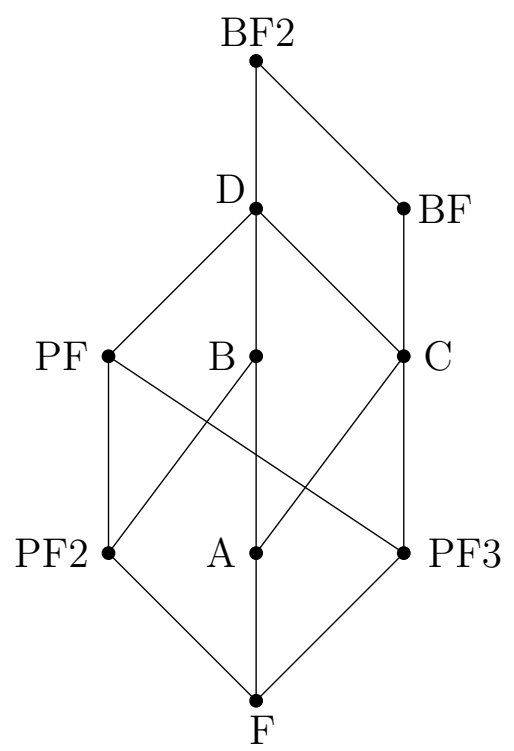

Fig. 7. The ten possibilities for a filter if positive implicativeness is also considered.

Pseudocomplementation filters are filters containing $\neg(x \sqcap \neg x)$ for all ${ }^{8} x$ in $L$. If the negation $\neg$ is involutive, then $\neg(x \sqcap \neg x)=x \sqcup \neg x$ and we can easily conclude that in this case Boolean filters coincide with pseudocomplementation filters. Note however that this sufficient condition is not a necessary condition. Indeed, consider the linear residuated lattice with four elements $0, a, b$, 1 determined by $x * x=0$ for all $x$ different from 1 . This residuated lattice does not have an involutive negation. Yet there is only one pseudocomplementation filter (namely $\{0, a, b, 1\}$ ), which is obviously also a Boolean filter. Also remark that not all pseudocomplementation filters are positive implicative filters. Indeed, consider the linear residuated lattice with four elements $0, a, b, 1(0<a<b<1)$ determined by $a * a=b * b=a$. Then $\{1\}$ is not a positive implicative filter $((b \sqcap(b \Rightarrow a)) \Rightarrow a=b \neq 1)$, although $\neg(0 \sqcap \neg 0)=\neg(a \sqcap \neg a)=\neg(b \sqcap \neg b)=\neg(1 \sqcap \neg 1)=1$.

Finally we also introduce involution filters. An involution filter of a residuated lattice $\mathcal{L}=(L, \sqcap, \sqcup, *, \Rightarrow, 0,1)$ is a filter of $\mathcal{L}$ that contains $\neg \neg x \Rightarrow x$ for every $x$ in $L$. Involution filters obviously satisfy the intersection and monotonicity property.

Proposition 17 Let $\mathcal{L}=(L, \sqcap, \sqcup, *, \Rightarrow, 0,1)$ be a residuated lattice and $F$ a filter of $\mathcal{L}$.

- If $F$ is a Boolean filter of $\mathcal{L}$, then $F$ is an involution filter of $\mathcal{L}$.

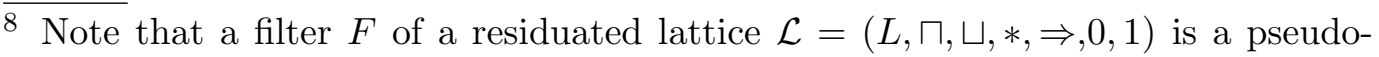
complementation filter of $\mathcal{L}$ iff $F$ contains $\neg(x \sqcap \neg x)$ for all $x$ in $\neg L$, or, equivalently, iff $F$ contains $\neg(\neg x \sqcap \neg \neg x)$ for all $x$ in $L$, because $\neg(\neg x \sqcap \neg \neg x) \leq \neg(x \sqcap \neg x)$. Also note that pseudocomplementation filters enjoy the intersection and monotonicity property. 
- If $F$ is a pseudocomplementation filter of $\mathcal{L}$ and at the same time an involution filter of $\mathcal{L}$, then $F$ is a Boolean filter of $\mathcal{L}$.

\section{PROOF.}

- Immediately, because $x \sqcup \neg x \leq \neg \neg x \Rightarrow x$ for all $x$ in $L$.

- Choose any $x$ in $L$. We know that $\neg \neg(x \sqcup \neg x) \Rightarrow(x \sqcup \neg x) \in F$. Because (using Proposition 1(10)) $\neg \neg(x \sqcup \neg x)=\neg(\neg x \sqcap \neg \neg x)=((\neg x) \sqcap(\neg x) \Rightarrow$ $0) \Rightarrow 0 \in F, x \sqcup \neg x \in F$.

\subsection{Congruence relations and quotient algebras}

Given a filter $F$ of a residuated lattice $\mathcal{L}=(L, \sqcap, \sqcup, *, \Rightarrow, 0,1)$, we can define the relation $\sim_{F}$ as follows: for all $x$ and $y$ in $L, x \sim_{F} y$ iff $x \Rightarrow y \in F$ and $y \Rightarrow x \in F$. This relation is a congruence on $\mathcal{L}$.

The congruence relations $\sim_{\{1\}}$ and $\sim_{L}$ are trivial: for all $x$ and $y$ in $L, x \sim_{\{1\}} y$ iff $x=y$; and $x \sim_{L} y$ always holds.

Based on the simple fact that $x \in F$ iff $[x]_{F}=[1]_{F}$, we immediately obtain the following properties, for every residuated lattice $\mathcal{L}=(L, \sqcap, \sqcup, *, \Rightarrow, 0,1)$ and filter $F$ of $\mathcal{L}$.

- The quotient algebra $\mathcal{L}_{\sim_{F}}$ is linear iff $F$ is a prime filter of $\mathcal{L}$.

- $[1]_{F}$ is $\sqcup_{F}$-irreducible in the quotient algebra $\mathcal{L}_{\sim_{F}}$ iff $F$ is a prime filter of the second kind of $\mathcal{L}$.

- The quotient algebra $\mathcal{L}_{\sim_{F}}$ is prelinear iff $F$ is a prime filter of the third kind of $\mathcal{L}$.

- The quotient algebra $\mathcal{L}_{\sim_{F}}$ is a Boolean algebra iff $F$ is a Boolean filter of $\mathcal{L}$.

- The quotient algebra $\mathcal{L}_{\sim_{F}}$ is a Boolean algebra with one or two elements iff $F$ is a Boolean filter of the second kind of $\mathcal{L}$.

- The quotient algebra $\mathcal{L}_{\sim_{F}}$ is a Heyting-algebra iff $F$ is a positive implicative filter of $\mathcal{L}$.

- The quotient algebra $\mathcal{L}_{\sim_{F}}$ has an involutive negation iff $F$ is an involution filter of $\mathcal{L}$.

\section{Interval-valued residuated lattices and triangle algebras}

Definition 18 Given a lattice $\mathcal{L}=(L, \sqcap, \sqcup)$, its triangularization $\mathbb{T}(\mathcal{L})$ is the structure $\mathbb{T}(\mathcal{L})=(\operatorname{Int}(\mathcal{L}), \sqcap, \sqcup)$ defined by 
- $\operatorname{Int}(\mathcal{L})=\left\{\left[x_{1}, x_{2}\right] \mid\left(x_{1}, x_{2}\right) \in L^{2}\right.$ and $\left.x_{1} \leq x_{2}\right\}$,

- $\left[x_{1}, x_{2}\right] \sqcap\left[y_{1}, y_{2}\right]=\left[x_{1} \sqcap y_{1}, x_{2} \sqcap y_{2}\right]$,

- $\left[x_{1}, x_{2}\right] \sqcup\left[y_{1}, y_{2}\right]=\left[x_{1} \sqcup y_{1}, x_{2} \sqcup y_{2}\right]$.

The set $D_{\mathcal{L}}=\{[x, x] \mid x \in L\}$ is called the diagonal of $\mathbb{T}(\mathcal{L})$.

Definition 19 An interval-valued residuated lattice (IVRL) is a residuated lattice $\left(\operatorname{Int}(\mathcal{L}), \sqcap, \sqcup, \odot, \Rightarrow_{\odot},[0,0],[1,1]\right)$ on the triangularization $\mathbb{T}(\mathcal{L})$ of a bounded lattice $\mathcal{L}$, in which the diagonal $D_{\mathcal{L}}$ is closed under $\odot$ and $\Rightarrow_{\odot}$, i.e., $[x, x] \odot[y, y] \in D_{\mathcal{L}}$ and $[x, x] \Rightarrow_{\odot}[y, y] \in D_{\mathcal{L}}$ for all $x, y$ in $L$.

In [26], we introduced the notion of triangle algebra, a structure that serves as an equational representation for an interval-valued residuated lattice.

Definition 20 A triangle algebra is a structure $\mathcal{A}=(A, \sqcap, \sqcup, *, \Rightarrow, \nu, \mu, 0, u, 1)$, in which $(A, \sqcap, \sqcup, *, \Rightarrow, 0,1)$ is a residuated lattice, $\nu$ and $\mu$ are unary operators on $A, u$ a constant, and satisfying the following conditions:

$$
\begin{array}{ll}
(T .1) \nu x \leq x, & \left(T .1^{\prime}\right) x \leq \mu x, \\
(T .2) \nu x \leq \nu \nu x, & \left(T .2^{\prime}\right) \mu \mu x \leq \mu x, \\
(T .3) \nu(x \sqcap y)=\nu x \sqcap \nu y, & \left(T .3^{\prime}\right) \mu(x \sqcap y)=\mu x \sqcap \mu y, \\
(T .4) \nu(x \sqcup y)=\nu x \sqcup \nu y, & \left(T .4^{\prime}\right) \mu(x \sqcup y)=\mu x \sqcup \mu y, \\
(T .5) \nu u=0, & \left(T .5^{\prime}\right) \mu u=1, \\
(T .6) \nu \mu x=\mu x, & \left(T .6^{\prime}\right) \mu \nu x=\nu x, \\
(T .7) \nu(x \Rightarrow y) \leq \nu x \Rightarrow \nu y, & \\
(T .8)(\nu x \Leftrightarrow \nu y) *(\mu x \Leftrightarrow \mu y) \leq(x \Leftrightarrow y), & \\
(T .9) \nu x \Rightarrow \nu y \leq \nu(\nu x \Rightarrow \nu y) . &
\end{array}
$$

In [26], we established a one-to-one correspondence between IVRLs and triangle algebras. It is shown in Figure 8. The unary operators $\nu$ and $\mu$ correspond with the projections $p_{v}$ and $p_{h}$ that map $\left[x_{1}, x_{2}\right]$ to $\left[x_{1}, x_{1}\right]$ and $\left[x_{2}, x_{2}\right]$ respectively. The constant $u$ corresponds to $[0,1]$. Theorem 21 gives this connection in more detail:

Theorem 21 [26] There is a one-to-one correspondence between the class of IVRLs and the class of triangle algebras. Every extended IVRL ${ }^{9}$ is a triangle

\footnotetext{
9 An extended IVRL is simply an IVRL in which the two mentioned projections are defined and the constant $[0,1]$ is fixed.
} 


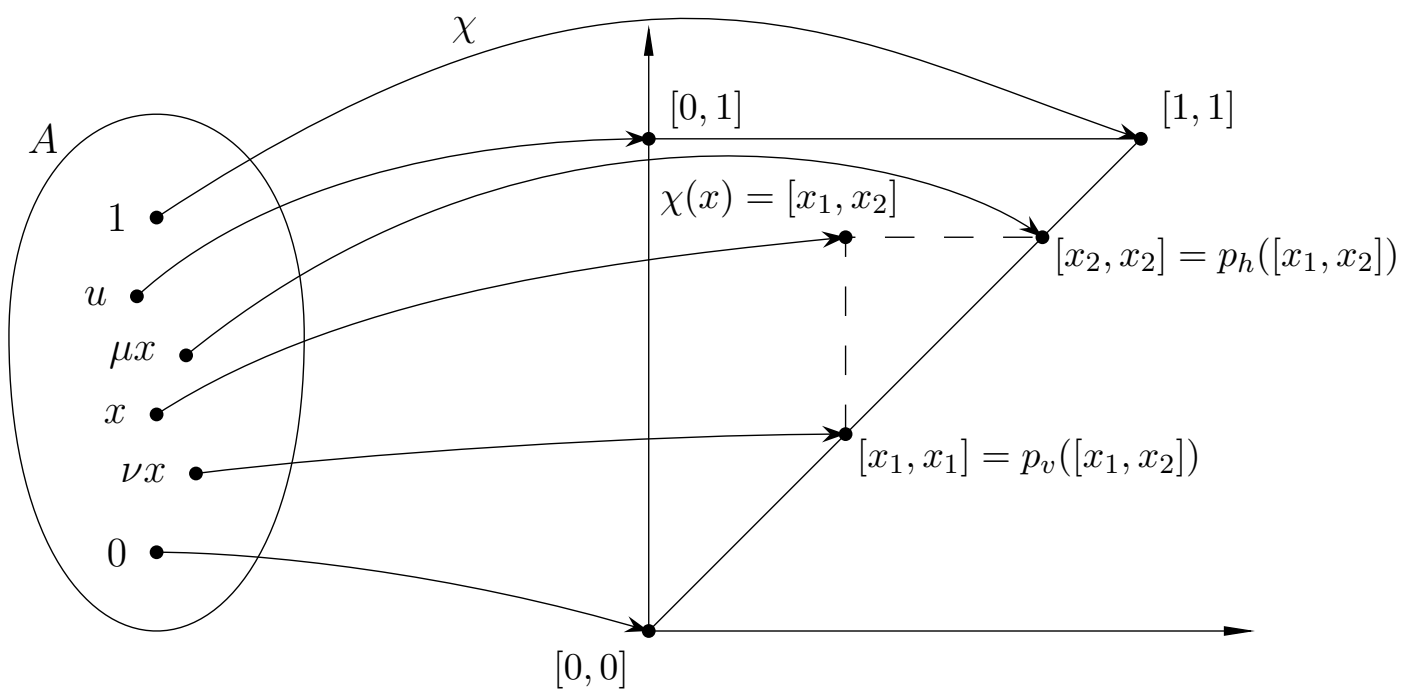

Triangle algebra

$(A, \sqcap, \sqcup, *, \Rightarrow, \nu, \mu, 0, u, 1)$

Isomorphic extended IVRL

$\left(A^{\prime}, \sqcap^{\prime}, \sqcup^{\prime}, *^{\prime}, \Rightarrow^{\prime}, p_{v}, p_{h},[0,0],[0,1],[1,1]\right)$

Fig. 8. The isomorphism $\chi$ from a triangle algebra to an IVRL.

algebra and conversely, every triangle algebra is isomorphic to an extended IVRL.

The idea behind interval degrees is that they provide a way to express incomplete as well as graded knowledge (see e.g. $[1,4,7,19,20,25,26]$ ). In fact, interval-valued fuzzy sets are a special case of type-2 fuzzy sets, which were introduced in [29].

Definition 22 [26] Let $\mathcal{A}=(A, \sqcap, \sqcup, *, \Rightarrow, \nu, \mu, 0, u, 1)$ be a triangle algebra. An element $x$ in $A$ is called exact if $\nu x=x$. The set of exact elements of $\mathcal{A}$ is denoted by $E(\mathcal{A})$.

Using the isomorphism in Figure 8, the set of exact elements of a triangle algebra corresponds to the diagonal of the isomorphic (extended) IVRL. In this paper we will sometimes use the term 'diagonal' for triangle algebras as well.

It was proven in $[26]$ that $E(\mathcal{A})$ is closed under all the defined operations on $\mathcal{A}$. We denote the subalgebra $(E(\mathcal{A}), \sqcap, \sqcup, *, \Rightarrow, 0,1)$ by $\mathcal{E}(\mathcal{A})$, which is a residuated lattice.

For any $x$ in a triangle algebra, it holds that $x=\nu x \sqcup(\mu x \sqcap u)$ (see [27]). Therefore, $x$ is completely determined by $\nu x$ and $\mu x$ (which are elements of $E(\mathcal{A}))$ : if $\nu x=\nu y$ and $\mu x=\mu y$, then $x=y$. 
In [27] we proved that

Theorem 23 In a triangle algebra $\mathcal{A}=(A, \sqcap, \sqcup, *, \Rightarrow, \nu, \mu, 0, u, 1)$, the implication $\Rightarrow$ and the product $*$ are completely determined by their action on $E(\mathcal{A})$ and the value of $\mu(u * u)$. More specifically:

- $\nu(x \Rightarrow y)=(\nu x \Rightarrow \nu y) \sqcap(\mu x \Rightarrow \mu y)$,

- $\mu(x \Rightarrow y)=(\mu x \Rightarrow(\mu(u * u) \Rightarrow \mu y)) \sqcap(\nu x \Rightarrow \mu y)$,

- $\nu(x * y)=\nu x * \nu y$,

- $\mu(x * y)=(\nu x * \mu y) \sqcup(\mu x * \nu y) \sqcup(\mu x * \mu y * \mu(u * u))$.

In [28], we introduced filters of triangle algebras. To clearly distinguish them from filters of residuated lattices, we will call them IVRL-filters in this paper.

Definition 24 Let $\mathcal{A}=(A, \sqcap, \sqcup, *, \Rightarrow, \nu, \mu, 0, u, 1)$ be a triangle algebra. An IVRL-filter (IF) of $\mathcal{A}$ is a non-empty subset $F$ of $A$, satisfying:

(F.1) if $x \in F, y \in A$ and $x \leq y$, then $y \in F$;

(F.2) if $x, y \in F$, then $x * y \in F$;

(F.3) if $x \in F$, then $\nu x \in F$.

For all $x$ and $y$ in $A$, we write $x \sim_{F} y$ iff $x \Rightarrow y$ and $y \Rightarrow x$ are both in $F$.

Remark that, because of (F.1), (F.3) and (T.1), we have

(F.3') $x \in F$ iff $\nu x \in F$.

Also remark that (F.2) and (F.3) can be replaced by "If $x, y \in F$, then $\nu(x *$ $y) \in F^{\prime \prime}$.

It was proven in [28] that $\sim_{F}$ is always a congruence relation. Note that (F.3) is a necessary condition for this statement. Indeed, if $\sim_{F}$ is a congruence relation on a triangle algebra $\mathcal{A}=(A, \sqcap, \sqcup, *, \Rightarrow, \nu, \mu, 0, u, 1)$ and $x \in F$, then $x \sim_{F} 1$ and therefore $\nu x \sim_{F} \nu 1=1$, which is equivalent with $\nu x \in F$.

\section{Filters of triangle algebras and their mutual connections}

There is an obvious connection between the notions 'IVRL-filter of a triangle algebra' and 'filter of a residuated lattice', which is given in the next proposition.

Proposition 25 Let $\mathcal{A}=(A, \sqcap, \sqcup, *, \Rightarrow, \nu, \mu, 0, u, 1)$ be a triangle algebra, $\mathcal{E}(\mathcal{A})=(E(\mathcal{A}), \sqcap, \sqcup, *, \Rightarrow, 0,1)$ be its subalgebra of exact elements and $F \subseteq A$. Then $F$ is a filter of the triangle algebra $\mathcal{A}$ iff (F.3') holds and $F \cap E(\mathcal{A})$ is a filter of the residuated lattice $\mathcal{E}(\mathcal{A})$.

The proof is straightforward.

Proposition 25 suggests two different ways to define specific kinds of IVRLfilters of triangle algebras. The first is to impose a property on a filter of 
the subalgabra of exact elements and extend this filter to the whole triangle algebra, using (F.3'). We call these IVRL-extended filters. For example, an IVRL-extended prime filter of the second kind (IPF2) of a triangle algebra $\mathcal{A}=(A, \sqcap, \sqcup, *, \Rightarrow, \nu, \mu, 0, u, 1)$ is a subset $F$ of $A$ such that $F \cap E(\mathcal{A})$ is a prime filter of the second kind of $\mathcal{E}(\mathcal{A})$ and $x \in F$ iff $\nu x \in F \cap E(\mathcal{A})$. Similarly we define IVRL-extended prime filters ${ }^{10}$ (IPF), IVRL-extended prime filters of the third kind (IPF3), IVRL-extended Boolean filters (IBF) and IVRLextended Boolean filters of the second kind (IBF2).

The second way is to impose a property on the whole IVRL-filter. For example, a Boolean IVRL-filter (BIF) of a triangle algebra $\mathcal{A}=(A, \sqcap, \sqcup, *, \Rightarrow$, $\nu, \mu, 0, u, 1)$ is an IVRL-filter $F$ of $A$ such that $F$ is a Boolean filter of $(A, \sqcap, \sqcup, *$, $\Rightarrow, 0,1)$. Similarly, we define prime IVRL-filters (PIF), prime IVRL-filters of the second kind (PIF2), prime IVRL-filters of the third kind (PIF3) and Boolean IVRL-filters of the second kind (BIF2). However, we will show that each of these is either trivial (the whole triangle algebra) or equivalent with an IVRL-extended filter.

We start with some properties that follow immediately from the definitions, Proposition 25 and the properties in the previous section.

Proposition 26 Let $\mathcal{A}=(A, \sqcap, \sqcup, *, \Rightarrow, \nu, \mu, 0, u, 1)$ be a triangle algebra and $F$ an IVRL-filter of $\mathcal{A}$.

(1) $A$ and $\{1\}$ are IVRL-filters of $\mathcal{A}$.

(2) $A$ is IPF, IPF2, IPF3, IBF, IBF2, PIF, PIF2, PIF3, BIF and BIF2.

(3) If $F$ is PIF, then $F$ is IPF.

(4) If $F$ is PIF2, then $F$ is IPF2.

(5) If $F$ is PIF3, then $F$ is IPF3.

(6) If $F$ is $B I F$, then $F$ is IBF.

(7) If $F$ is BIF2, then $F$ is IBF2.

(8) The intersection property holds for IF, IPF3, IBF, PIF3 and BIF (but not for IPF, IPF2 or IBF2).

(9) The monotonicity property holds for IF, IPF, IPF3, IBF, IBF2, PIF, PIF3, BIF and BIF2 (but not for IPF2 or PIF2).

(10) The implications and equivalences from the previous section can be 'translated' to the two kinds of IVRL-filters. For example: $F$ is IPF iff $F$ is IPF2 and IPF3, and F is PIF iff F is PIF2 and PIF3.

(11) The counterexamples from the previous section can be 'translated' to the IVRL-extended filters. For example: $F$ can be IBF without being IPF2.

\footnotetext{
$\left.\overline{{ }^{10} \mathrm{In}[28}\right]$, we called these pseudo-prime filters. They were used to prove that triangle algebras with a prelinear subalgebra of exact elements are subdirect products of triangle algebras with a linear subalgebra of exact elements.
} 
Boolean IVRL-filters and Boolean IVRL-filters of the second kind are trivial. This is a consequence of the following proposition.

Proposition 27 Let $\mathcal{A}=(A, \sqcap, \sqcup, *, \Rightarrow, \nu, \mu, 0, u, 1)$ be a triangle algebra and $F$ a Boolean IVRL-filter of $\mathcal{A}$. Then $F=A$.

PROOF. Because $u \sqcup \neg u$ must be in $F$ and therefore also $\nu(u \sqcup \neg u)=$ $\nu u \sqcup \nu \neg u=0 \sqcup \neg \mu u=\neg 1=0$. That $\nu \neg u=\neg \mu u$ is a consequence of Theorem 23.

In Proposition 26(4) we already saw that prime IVRL-filters of the second kind are IVRL-extended prime filters of the second kind. Now we show the converse holds as well.

Proposition 28 Let $\mathcal{A}=(A, \sqcap, \sqcup, *, \Rightarrow, \nu, \mu, 0, u, 1)$ be a triangle algebra and $F$ an IVRL-extended prime filter of the second kind of $\mathcal{A}$. Then $F$ is a prime IVRL-filter of the second kind of $\mathcal{A}$.

PROOF. Suppose $x \sqcup y \in F$. Then also $\nu x \sqcup \nu y=\nu(x \sqcup y) \in F$. Because $\nu x$ and $\nu y$ are exact elements and $F$ is an IVRL-extended prime filter of the second kind of $\mathcal{A}, \nu x$ or $\nu y$ must be in $F$. This implies that $F$ contains $x$ or $y$. We conclude $F$ must be a prime IVRL-filter of the second kind of $\mathcal{A}$.

Now we show that prime IVRL-filters of the third kind are the same as IVRLextended Boolean filters.

Proposition 29 Let $\mathcal{A}=(A, \sqcap, \sqcup, *, \Rightarrow, \nu, \mu, 0, u, 1)$ be a triangle algebra and $F$ a subset of $A$. Then the following two statements are equivalent:

- F is a prime IVRL-filter of the third kind of $\mathcal{A}$,

- $F$ is an IVRL-extended Boolean filter of $\mathcal{A}$.

PROOF. Suppose $F$ is a prime IVRL-filter of the third kind of $\mathcal{A}$ and choose an arbitrary exact element $\nu x$. We want to show that $\nu x \sqcup \neg \nu x \in F$. Indeed, we know $(u \Rightarrow \nu x) \sqcup(\nu x \Rightarrow u) \in F$ and therefore also

$$
\begin{aligned}
\nu x \sqcup \neg \nu x & =((\nu u \Rightarrow \nu \nu x) \sqcap(\mu u \Rightarrow \mu \nu x)) \sqcup((\nu \nu x \Rightarrow \nu u) \sqcap(\mu \nu x \Rightarrow \mu u)) \\
& =\nu(u \Rightarrow \nu x) \sqcup \nu(\nu x \Rightarrow u) \\
& =\nu((u \Rightarrow \nu x) \sqcup(\nu x \Rightarrow u)) \in F .
\end{aligned}
$$

Conversely, suppose $F$ is an IVRL-extended Boolean filter of $\mathcal{A}$. Take any two elements $x$ and $y$ in $A$. We have to show that $(x \Rightarrow y) \sqcup(y \Rightarrow x) \in F$. 
First note that $F$ contains $\nu x \sqcup \neg \nu x, \mu x \sqcup \neg \mu x, \nu y \sqcup \neg \nu y$ and $\mu y \sqcup \neg \mu y$, and therefore also $S:=(\nu x \sqcup \neg \nu x) *(\mu x \sqcup \neg \mu x) *(\nu y \sqcup \neg \nu y) *(\mu y \sqcup \neg \mu y)$. Using the properties $a *(b \sqcup c)=(a * b) \sqcup(a * c)$ and $d * e \leq d \sqcap e$, we can easily see that $S \leq \nu x \sqcup \neg \mu x \sqcup \nu y \sqcup \neg \mu y \sqcup(\neg \nu x \sqcap \mu x \sqcap \neg \nu y \sqcap \mu y)$. Indeed, $S$ is the supremum of all elements of the form $f * g * h * i$, with $f \in\{\nu x, \neg \nu x\}, g \in\{\mu x, \neg \mu x\}$, $h \in\{\nu y, \neg \nu y\}$ and $i \in\{\mu y, \neg \mu y\}$. Apart from $\neg \nu x * \mu x * \neg \nu y * \mu y$, any of these elements is smaller than or equal to $\nu x \sqcup \neg \mu x \sqcup \nu y \sqcup \neg \mu y$.

So, using $\neg x \sqcup y \leq x \Rightarrow y$, and $\nu y=\nu y \sqcup(\nu y \sqcap a)=(\nu y \sqcap \mu y) \sqcup(\nu y \sqcap a)$, for all $x, y$ and $a$ in $A$, we find

$$
\begin{aligned}
S \leq & \neg \mu x \sqcup(\neg \nu x \sqcap \mu y) \sqcup \nu y \sqcup \neg \mu y \sqcup(\neg \nu y \sqcap \mu x) \sqcup \nu x \\
= & (\neg \nu x \sqcap \neg \mu x) \sqcup(\neg \nu x \sqcap \mu y) \sqcup(\nu y \sqcap \neg \mu x) \sqcup(\nu y \sqcap \mu y) \sqcup \\
& (\neg \nu y \sqcap \neg \mu y) \sqcup(\neg \nu y \sqcap \mu x) \sqcup(\nu x \sqcap \neg \mu y) \sqcup(\nu x \sqcap \mu x) \\
\leq & ((\neg \nu x \sqcup \nu y) \sqcap(\neg \mu x \sqcup \mu y)) \sqcup((\neg \nu y \sqcup \nu x) \sqcap(\neg \mu y \sqcup \mu x)) \\
\leq & ((\nu x \Rightarrow \nu y) \sqcap(\mu x \Rightarrow \mu y)) \sqcup((\nu y \Rightarrow \nu x) \sqcap(\mu y \Rightarrow \mu x)) \\
= & \nu((x \Rightarrow y) \sqcup(y \Rightarrow x)) \\
\leq & (x \Rightarrow y) \sqcup(y \Rightarrow x),
\end{aligned}
$$

which implies $(x \Rightarrow y) \sqcup(y \Rightarrow x) \in F$.

Remark that Proposition 29 generalizes a result from [28] (Proposition 18), namely that a triangle algebra is prelinear iff its subalgebra of exact elements is a Boolean algebra. Indeed, this is exactly Proposition 29 applied to $F=\{1\}$.

Corollary 30 Let $\mathcal{A}=(A, \sqcap, \sqcup, *, \Rightarrow, \nu, \mu, 0, u, 1)$ be a triangle algebra and $F$ a subset of $A$. Then the following two statements are equivalent:

- $F$ is a prime IVRL-filter of $\mathcal{A}$,

- $F$ is an IVRL-extended Boolean filter of the second kind of $\mathcal{A}$.

PROOF. Remark that $F$ is a prime IVRL-filter of $\mathcal{A}$ iff $F$ is a prime IVRLfilter of the second kind of $\mathcal{A}$ and a prime IVRL-filter of the third kind of $\mathcal{A}$. Then note that $F$ is an IVRL-extended Boolean filter of the second kind of $\mathcal{A}$ iff $F$ is an IVRL-extended prime filter of the second kind of $\mathcal{A}$ and $F$ an IVRL-extended Boolean filter of $\mathcal{A}$.

Therefore the result follows immediately from Propositions 28 and 29.

Choosing $F=\{1\}$ gives: a triangle algebra is linear iff its subalgebra of exact elements has one or two elements. 


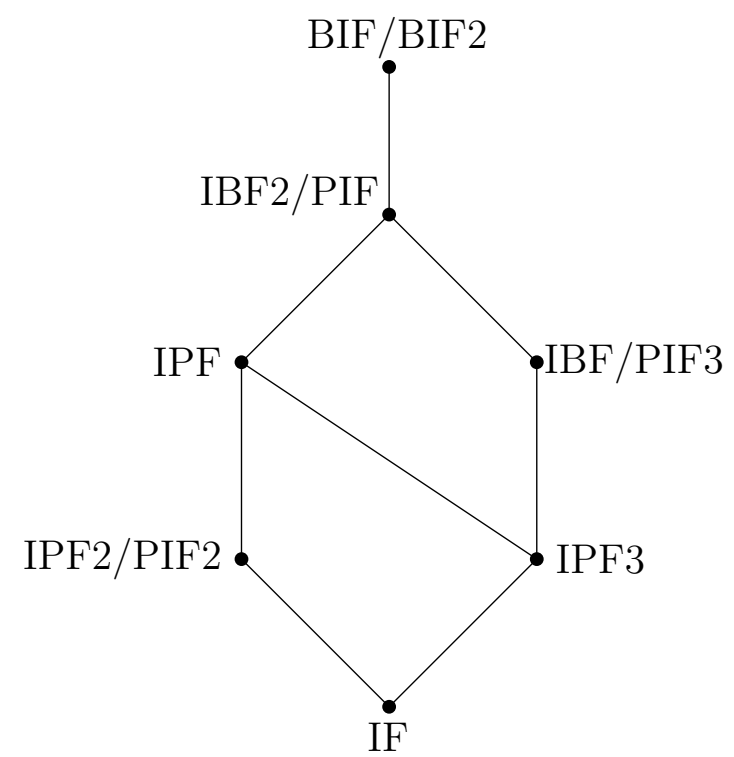

Fig. 9. The seven possibilities for an IVRL-filter.

Similarly as for filters of residuated lattices, we can summarize the different possibilities for an IVRL-filter $F$ of a triangle algebra $\mathcal{A}=(A, \sqcap, \sqcup, *, \Rightarrow$, $\nu, \mu, 0, u, 1)$ :

- $F$ is an IVRL-filter of $\mathcal{A}$, but $F$ is not IPF, PIF, IPF2, PIF2, IPF3, PIF3, IBF, BIF, IBF2 nor BIF2;

- $F$ is IPF2 (equivalently, PIF2), but not IPF, PIF, IPF3, PIF3, IBF, BIF, IBF2 nor BIF2;

- $F$ is IPF3, but not IPF, PIF, IPF2, PIF2, PIF3, IBF, BIF, IBF2 nor BIF2;

- $F$ is IPF (and therefore also IPF2/PIF2 and IPF3), but not PIF, PIF3, IBF, BIF, IBF2 nor BIF2;

- $F$ is IBF (equivalently, PIF3) (and therefore also IPF3), but not IPF, PIF, IPF2, PIF2, IBF2, BIF nor BIF2;

- $F$ is IBF2 (equivalently, PIF) (and therefore also IPF, IPF2, PIF2, IPF3, PIF3 and IBF), but not BIF nor BIF2;

- $F=A$, in this trivial case, $F$ is IPF, PIF, IPF2, PIF2, IPF3, PIF3, IBF, BIF, IBF2 and BIF2.

The lattice in Figure 9 gives a schematic summary of these situations. Remark that the IVRL-extended filters suffice to cover all six non-trivial cases. Therefore the scheme looks the same as for residuated lattices.

Remark 31 Because of Theorem 23 a triangle algebra $\mathcal{A}=(A, \sqcap, \sqcup, *, \Rightarrow$ $, \nu, \mu, 0, u, 1)$ is completely determined by the value $\mu(u * u)$ and its subalgebra $\mathcal{E}(\mathcal{A})$ of exact elements. It is quite remarkable that for the filters shown in Figure 9, the value $\mu(u * u)$ is not important. For example, if $F$ is a prime filter of a triangle algebra $\mathcal{A}$ with $\mathcal{E}(\mathcal{A})=\mathcal{L}$, then $F$ is also a prime filter of every triangle algebra with $\mathcal{L}$ as subalgebra of exact elements.

For other kinds of filters this does not necessarily hold. We will show this for positive implicative IVRL-filters, pseudocomplementation IVRL-filters and 
involution IVRL-filters. In the proofs we will use the residuation principle, the monotonicity of the operations and Theorem 23 without always mentioning it. The same goes for easy properties such as $\nu x \leq x \leq \mu x, x \Rightarrow y=1$ iff $x \leq y$, $1 \Rightarrow x=x, \ldots$

Proposition 32 Let $\mathcal{A}=(A, \sqcap, \sqcup, *, \Rightarrow, \nu, \mu, 0, u, 1)$ be a triangle algebra and $F \subseteq A$. Then $F$ is a positive implicative IVRL-filter of $\mathcal{A}$ iff $F$ is an IVRLextended positive implicative filter of $\mathcal{A}$ and $\mu(u * u) \in F$.

PROOF. First remark that an IVRL-filter $F$ of a triangle algebra $\mathcal{A}=$ $(A, \sqcap, \sqcup, *, \Rightarrow, \nu, \mu, 0, u, 1)$ is a positive implicative IVRL-filter iff it contains $\nu(x \Rightarrow(x * x))=(\nu x \Rightarrow(\nu x * \nu x)) \sqcap(\mu x \Rightarrow((\mu x * \nu x) \sqcup(\mu x * \mu x * \mu(u * u))))$ for all $x$ in $A$. Taking $x=u$, we see that $\mu(u * u)$ must be in $F$. Obviously $F$ must also be an IVRL-extended positive implicative filter of $\mathcal{A}$.

These two conditions are also sufficient, as they imply $\nu x \Rightarrow(\nu x * \nu x) \in F$, $\mu x \Rightarrow(\mu x * \mu x) \in F$ and $\mu(u * u) \in F$, such that also $\nu(x \Rightarrow(x * x)) \in F$ because (using Proposition 1(8 and 1)) $(\nu x \Rightarrow(\nu x * \nu x)) *(\mu x \Rightarrow(\mu x * \mu x)) * \mu(u * u) \leq$ $(\nu x \Rightarrow(\nu x * \nu x)) *(\mu x \Rightarrow((\mu x * \mu x) * \mu(u * u))) \leq \nu(x \Rightarrow(x * x))$.

Proposition 33 Let $\mathcal{A}=(A, \sqcap, \sqcup, *, \Rightarrow, \nu, \mu, 0, u, 1)$ be a triangle algebra and $F \subseteq A$. Then $F$ is a pseudocomplementation IVRL-filter of $\mathcal{A}$ iff $F$ is an IVRL-extended pseudocomplementation filter of $\mathcal{A}$ and $\neg \neg \mu(u * u) \in F$.

PROOF. An IVRL-filter $F$ of a triangle algebra $\mathcal{A}=(A, \sqcap, \sqcup, *, \Rightarrow, \nu, \mu, 0, u$, $1)$ is a pseudocomplementation IVRL-filter iff it contains $\nu(\neg(x \sqcap \neg x))=$ $\neg(\nu x \sqcap \neg \mu x) \sqcap \neg(\mu x \sqcap \neg(\mu x * \mu(u * u)) \sqcap \neg \nu x)$ for all $x$ in $A$. Taking $x=u$, we see that $\neg \neg \mu(u * u)$ must be in $F$. Obviously $F$ must also be an IVRLextended pseudocomplementation filter of $\mathcal{A}$.

These two conditions are also sufficient. To see this, choose any $x$ in $A$, denote $\mu x \sqcap \neg(\mu x * \mu(u * u))$ by $X$ and remark that (using Proposition $1(11,7,2$ and $9))$

$$
\begin{aligned}
X & =\mu x \sqcap \neg(\mu x * \mu(u * u)) \\
& =\mu x \sqcap(\mu x \Rightarrow \neg \mu(u * u)) \\
& =\mu x \sqcap(\mu x \Rightarrow \neg \neg \neg \mu(u * u)) \\
& =\mu x \sqcap \neg(\mu x * \neg \neg \mu(u * u)) \\
& =\mu x \sqcap(\neg \neg \mu(u * u) \Rightarrow \neg \mu x) \\
& \leq(\neg \neg \mu(u * u) \Rightarrow \mu x) \sqcap(\neg \neg \mu(u * u) \Rightarrow \neg \mu x) \\
& =\neg \neg \mu(u * u) \Rightarrow(\mu x \sqcap \neg \mu x) .
\end{aligned}
$$


This implies (using Proposition 1(5))

$$
\begin{aligned}
& \neg \neg \mu(u * u) * X * \neg(\mu x \sqcap \neg \mu x) \\
& \leq \neg \neg \mu(u * u) *(\neg \neg \mu(u * u) \Rightarrow(\mu x \sqcap \neg \mu x)) * \neg(\mu x \sqcap \neg \mu x) \\
& \leq(\mu x \sqcap \neg \mu x) * \neg(\mu x \sqcap \neg \mu x) \\
& =0 .
\end{aligned}
$$

Therefore, $\neg \neg \mu(u * u) * \neg(\mu x \sqcap \neg \mu x) \leq \neg X$, so $\neg X$ must be in $F$. This concludes the proof because $\neg(\mu x \sqcap \neg \mu x) \sqcap \neg X \leq \nu(\neg(x \sqcap \neg x))$.

Proposition 34 Let $\mathcal{A}=(A, \sqcap, \sqcup, *, \Rightarrow, \nu, \mu, 0, u, 1)$ be a triangle algebra and $F \subseteq A$. Then $F$ is an involution IVRL-filter of $\mathcal{A}$ iff $F$ is an IVRL-extended involution filter of $\mathcal{A}$ and $\neg \mu(u * u) \in F$.

PROOF. Remark that an IVRL-filter $F$ of a triangle algebra $\mathcal{A}=(A, \sqcap, \sqcup, *$, $\Rightarrow, \nu, \mu, 0, u, 1)$ is an involution IVRL-filter iff it contains

$$
\begin{aligned}
\nu(\neg \neg x \Rightarrow x)= & (\neg(\neg(\mu x * \mu(u * u)) \sqcap \neg \nu x) \Rightarrow \nu x) \\
& \sqcap((\neg((\neg(\mu x * \mu(u * u)) \sqcap \neg \nu x) * \mu(u * u)) \sqcap \neg \neg \mu x) \Rightarrow \mu x)
\end{aligned}
$$

for all $x$ in $A$. Taking $x=u$, we see that $\neg \neg \neg \mu(u * u)=\neg \mu(u * u)$ must be in $F$. Obviously $F$ must also be an IVRL-extended involution filter of $\mathcal{A}$.

These two conditions are also sufficient. Indeed, as $\nu x \leq \mu x$, it follows that

$$
\begin{aligned}
& (\neg((\neg(\mu x * \mu(u * u)) \sqcap \neg \nu x) * \mu(u * u)) \sqcap \neg \neg \mu x) \Rightarrow \mu x \\
& \geq(\neg((\neg(\mu x * \mu(u * u)) \sqcap \neg \mu x) * \mu(u * u)) \sqcap \neg \neg \mu x) \Rightarrow \mu x \\
& =\neg \neg \mu x \Rightarrow \mu x \in F .
\end{aligned}
$$

Furthermore, using Proposition 1(3, 9 and 2), we find

$$
\begin{aligned}
& \neg \mu(u * u) * \neg(\neg \mu(u * u) \sqcap \neg \nu x) \\
& \leq \neg(\neg \mu(u * u) \Rightarrow(\neg \mu(u * u) \sqcap \neg \nu x)) \\
& =\neg(\neg \mu(u * u) \Rightarrow \neg \nu x) \\
& \leq \neg \neg \nu x,
\end{aligned}
$$

such that (using Proposition 1(5))

$$
\begin{aligned}
& \neg \mu(u * u) * \neg(\neg \mu(u * u) \sqcap \neg \nu x) *(\neg \neg \nu x \Rightarrow \nu x) \\
& \leq \neg \neg \nu x *(\neg \neg \nu x \Rightarrow \nu x) \\
& \leq \nu x
\end{aligned}
$$


and therefore

$$
\begin{aligned}
& \neg(\neg(\mu x * \mu(u * u)) \sqcap \neg \nu x) \Rightarrow \nu x \\
& \geq \neg(\neg(1 * \mu(u * u)) \sqcap \neg \nu x) \Rightarrow \nu x \\
& \geq \neg \mu(u * u) *(\neg \neg \nu x \Rightarrow \nu x) \in F .
\end{aligned}
$$

Remark that this result is a generalization of Corollary 16 in [27]. Indeed, we obtain this property by choosing $F=\{1\}$ in Proposition 34 .

Example 35 An easy example to illustrate the importance of the value $\mu(u *$ u) for positive implicative, pseudocomplementation and involution filters of triangle algebras, is the following. Consider the two triangle algebras with three elements. One of them is determined by $u * u=0$, the other by $u * u=u$. In both cases, the subalgebra of exact elements is a Boolean algebra (it has two elements). Therefore $\{1\}$ is IBF (but not BIF). In the first triangle algebra (determined by $\mu(u * u)=0),\{1\}$ is an involution IVRL-filter, but not a pseudocomplementation IVRL-filter (and therefore not a positive implicative IVRL-filter). In the second triangle algebra (determined by $\mu(u * u)=1$ ), it is the other way around: $\{1\}$ is a positive implicative IVRL-filter (and therefore also a pseudocomplementation IVRL-filter), but not an involution IVRL-filter.

\section{Conclusion and future work}

In this paper we introduced different kinds of filters of residuated lattices, such as prime filters of the third kind, Boolean filters of the second kind and involution filters. We gave alternative definitions for positive implicative filters. We gave several examples of and investigated the connections between these different kinds of filters. We also found a characterization of finite MTLalgebras in terms of filters. Whether this characterization is also valid for infinite MTL-algebras is still an open problem. Then we showed that the definitions of the different kinds of filters of residuated lattices can be extended to triangle algebras in two different ways. We examined the relationships between the obtained definitions. Some of these relationships are generalizations of already known theorems. The investigation of other such generalizations can be an interesting topic for further research. 


\section{References}

[1] C. Cornelis, G. Deschrijver, E.E. Kerre, Advances and challenges in interval-valued fuzzy logic, Fuzzy Sets and Systems 157(5), (2006), 622627

[2] R.P. Dilworth and M. Ward, Residuated Lattices, Trans. A.M.S. 45, (1939), $335-354$

[3] M. Dummett, A propositional calculus with denumerable matrix, The Journal of Symbolic Logic 24(2), (1959), 97-106

[4] F. Esteva, P. Garcia-Calvés, L. Godo, Enriched Interval Bilattices and Partial Many-Valued Logics: an Approach to Deal with Graded Truth and Imprecision, International Journal of Uncertainty, Fuzziness and Knowledge-Based Systems, Vol. 2(1), (1994), 37-54

[5] F. Esteva, L. Godo, Monoidal t-norm Based Logic: Towards a Logic for Left-Continuous t-norms, Fuzzy Sets and Systems 124, (2001), 271-288

[6] F. Esteva, L. Godo, A. Garcia-Cerdaña, On the Hierarchy of t-norm Based Residuated Fuzzy Logics, in: Beyond Two: Theory and Applications of Multiple Valued Logic (M. Fitting and E. Orlowska, eds.), Physica-Verlag, (2003), 251-272

[7] M. Gehrke, C. Walker, E. Walker, Some comments on interval-valued fuzzy sets, International Journal of Intelligent Systems 11, (1996), 751-759

[8] K. Gödel, Zum intuitionistischen Aussagenkalkül, Anzeiger der Akademie der Wissenschaften in Wien, (1932), 65-66

[9] S. Gottwald, Mathematical fuzzy logic as a tool for the treatment of vague information, Information Sciences 172, (2005), 41-71

[10] P. Hájek, Metamathematics of Fuzzy Logic, Trends in Logic-Studia Logica Library, Kluwer Academic Publishers, (1998)

[11] A. Heyting, Die formalen Regeln der intuitionistischen Logik, Sitzungsberichte der preuszischen Akademie der Wissenschaften, physikalischmathematische Klasse, (1930), 42-56 57-71 158-169 in three parts, Sitzungsber. preuss. Akad. Wiss.: 42-71, 158-169. English translation of Part I in Mancosu 1998: 311-327.

[12] U. Höhle, Commutative, Residuated l-monoids, in: Non-classical Logics and their Applications to Fuzzy Subsets: a Handbook of the Mathematical Foundations of Fuzzy Set Theory (U. Höhle and E.P. Klement, eds.), Kluwer Academic Publishers, (1995), 53-106

[13] E.V. Huntington, Sets of independent postulates for the algebra of logic, Trans. A.M.S. 5, (1904), 288-309 
[14] P. Jipsen, Some lists of finite structures, at http://www1.chapman.edu/ jipsen/gap/rl.html,

[15] Y.B. Jun, Y. Xu, X.H. Zhang, Fuzzy filters of MTL-algebras, Information Sciences 175, (2005), 120-138

[16] L. Liu, K. Li, Fuzzy filters of BL-algebras, Information Sciences 173, (2005), $141-154$

[17] L. Liu, K. Li, Boolean filters and positive implicative filters of residuated lattices, Information Sciences 177(24), (2007), 5725-5738

[18] J. Łukasiewicz, A. Tarski, Untersuchungen über den Aussagenkalkül, Comptes Rendus de la Société des Sciences et des Lettres de Varsovie, (1930), $1-21$

[19] J.M. Mendel, Uncertain Rule-Based Fuzzy Logic Systems, Prentice Hall PTR, Upper Saddle River, New Jersey, (2001)

[20] J.M. Mendel, Advances in type-2 fuzzy sets and systems, Information Sciences 177, (2007), 84-110

[21] J. Rachunek, D. Salounová, Fuzzy filters and fuzzy prime filters of bounded Rl-monoids and pseudo BL-algebras, Information Sciences 178(17), (2008), 3474-3481

[22] H. Rasiowa, R. Sikorski, The mathematics of metamathematics, Polska Akad. Nauk, (1963)

[23] E. Turunen, Mathematics behind Fuzzy Logic, Physica-Verlag, (1999)

[24] E. Turunen, Boolean deductive systems of BL-algebras, Archive for Mathematical Logic 40, (2001), 467-473

[25] B. Van Gasse, C. Cornelis, G. Deschrijver, E.E. Kerre, On the properties of a generalized class of t-norms in interval-valued fuzzy logics, New Mathematics and Natural Computation, Vol. 2 (No. 1), (2006), 29-42

[26] B. Van Gasse, C. Cornelis, G. Deschrijver, E.E. Kerre, Triangle algebras: A formal logic approach to interval-valued residuated lattices, Fuzzy Sets and Systems 159, (2008), 1042-1060

[27] B. Van Gasse, C. Cornelis, G. Deschrijver, E.E. Kerre, A characterization of interval-valued residuated lattices, International Journal of Approximate Reasoning 49, (2008), 478-487

[28] B. Van Gasse, C. Cornelis, G. Deschrijver, E.E. Kerre, The pseudo-linear semantics of interval-valued fuzzy logics, Information Sciences 179, (2009), $717-728$

[29] L.A. Zadeh, The concept of a linguistic variable and its application to approximate reasoning - I, Information Sciences 8, (1975), 199-249 